\title{
Caracterización clínica de pacientes con recurrencia de úlcera por enfermedad venosa crónica.
}

\section{Characteristics of patients with recurrence of ulcer due to chronic venous insufficiency in patients.}

\author{
Víctor Augusto Beltrán Guaqueta ${ }^{1,2}$; Adriana Patricia Bulla Silva ${ }^{2}$; Nataly Johana Espitia Suarez ${ }^{2}$; \\ Diana Carolina Vargas Quintero ${ }^{2}$; Ledmar Jovanny Vargas Rodríguez ${ }^{3}{ }^{*}$. \\ Departamento de cirugía general, Hospital San Rafael. Tunja, Colombia. \\ 2 Grupo de clínica de heridas, Hospital San Rafael. Tunja, Colombia. \\ 3 Departamento de investigación, Hospital San Rafael, Universidad de Boyacá, Facultad de ciencias de la salud. Tunja, \\ Colombia. \\ *Dirigir correspondencia a lejovaro@gmail.com
}

Proceso Editorial

Recibido: 160820

Aceptado: 151220

Publicado: 230321

DOI 10.17081/innosa.118

CCopyright 2021.

Beltrán 1 et al.

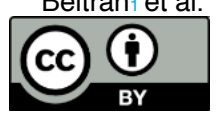

\section{RESUMEN}

Introducción: La enfermedad venosa crónica es una patología derivada de alteraciones anatómicas o funcionales del sistema venoso. El objetivo de este estudio era determinar las características de los pacientes con recurrencia de úlcera por enfermedad venosa crónica en usuarios del programa clínica de heridas de un hospital de tercer nivel de Tunja. Métodos: Se realizó un estudio descriptivo, retrospectivo y de corte transversal en el periodo 2017 a 2019 , donde se incluyeron pacientes mayores de 18 años de edad con diagnostico ulcera vascular por enfermedad venosa crónica. Resultados: La prevalencia de recurrencia fue de 15.7\%. La media de edad de los participantes fue de 65 años, donde el $72.7 \%$ eran mujeres y el $77.3 \%$ de los participantes vivían solos. La hipertensión (72,7\%) y el sobrepeso $(45,5 \%)$ fueron las comorbilidades que se presentaban con mayor frecuencia. La extremidad inferior más afectada fue la izquierda con $68.2 \%$. Las personas permanecían 50.9 minutos al día con la extremidad elevada y 6.6 horas al día en bipedestación. Conclusiones: La prevalencia de recurrencia de úlcera por enfermedad venosa crónica en el presente estudio es menor a la reportada en la literatura, donde se ve afectada la población femenina, con 65 años de edad, asociado a múltiples comorbilidades como el sobrepeso, la desnutrición, el tabaquismo, la dislipidemia, entre otras. Afecta con mayor frecuencia el miembro inferior izquierdo y los manejos más utilizados son los quirúrgicos (varicosafenectomía) y la terapia compresiva a presión media y alta. A partir de este estudio se invita a otras instituciones a evaluar los programas de manejo de heridas con el fin de analizar el impacto y conocer este grupo poblacional.

Palabras clave: Úlcera de la Pierna; Úlcera Cutánea; Úlcera Varicosa; Enfermedad Venosa; Recurrencia (DeCS).

\section{ABSTRACT}

Background: Chronic venous disease is a pathology derived from anatomical or functional alterations of the venous system. The objective of this study was to determine the characteristics of patients with recurrence of ulcer due to chronic venous disease in users of the clinical wound program of a tertiary hospital in Tunja. Methods: A descriptive, retrospective and cross-sectional study was carried out in the period 2017 to 2019, which included patients older than 18 years of age with a diagnosis of vascular ulcer due to chronic venous disease. Results: The prevalence of recurrence was $15.7 \%$. The mean age of the participants was 65 years, where $72.7 \%$ were women and $77.3 \%$ of the participants lived alone. Hypertension $(72.7 \%)$ and overweight $(45.5 \%)$ were the comorbidities that occurred with the highest frequency. The lower limb most affected was the left with $68.2 \%$. People spent 50.9 minutes a day with the limb elevated and 6.6 hours a day in a standing position. Conclusions: The prevalence of ulcer recurrence due to chronic venous disease in the present study is lower than that reported in the literature, where the female population, 65 years of age, is affected, associated with multiple comorbidities such as overweight, malnutrition, smoking, dyslipidemia, among others. It most frequently affects the lower left limb and the most commonly used procedures are surgical (varicosafenectomy) and medium and high pressure compression therapy. Based on this study, other institutions are invited to evaluate wound management programs in order to analyze the impact and learn about this population group.

Keywords: Leg Ulcer; Skin Ulcer; Varicose Ulcer; Venous Insufficiency; Recurrence (MeSH). 


\section{INTRODUCCIÓN}

Las ulceras vasculares tienen una incidencia que varía entre el 8 al $15 \%$ en los pacientes mayores de 65 años, generando importante repercusiones en términos sociales y económicos por los costos elevados para los sistemas de salud y para su entorno, además que afecta la calidad de vida del paciente (1).

La enfermedad venosa crónica es una patología derivada de alteraciones anatómicas o funcionales del sistema venoso (2), donde se presenta un desequilibrio a nivel de la microcirculación que incluyen hipertensión venosa secundaria, incompetencia valvular, entre otros factores como el sedentarismo, el ortostatismo y la bipedestación que disminuye la función de la bomba muscular, dificultando el retorno venoso y genera un aumento de la presión en estos vasos $(\underline{3}, \underline{4})$.

La enfermedad venosa crónica es una de las patologías más frecuentes, con una prevalencia entre 25 a 30\% en la población adulta; Además, es importante tener en cuenta que la prevalencia esta entidad aumenta linealmente con la edad $(\underline{5}, \underline{6}, \underline{7})$. Esta entidad patológica se puede clasificar mediante el CEAP (acrónimo de Clínica, Etiología, Anatomía y Patofisiología) que es una escala aceptada a nivel global, por medio de la cual se facilita la descripción de las formas de enfermedad venosa crónica, sin embargo se debe aclarar que dicha clasificación no permite conocer el impacto de la enfermedad venosa crónica en el paciente ni la calidad de vida $(\underline{5}, \underline{8})$ (Tabla 1).

La recurrencia o recidiva se define como la reaparición de la enfermedad tras un período de ausencia. Las ulceras venosas presentan una tasa de recurrencia a los 12 meses que varía del $26 \%$ al $69 \%(\underline{4}, \underline{7}, \underline{8}, \underline{9})$, fue por este motivo que se decidió realizar el presente estudio el cual tiene como objetivo conocer las características de los pacientes con recurrencia de úlcera por enfermedad venosa crónica en usuarios del programa clínica de heridas de un hospital de tercer nivel de Tunja, puesto que esto facilitara tener presente que pacientes atendidos por el programa puedan presentar este tipo de complicaciones.

\section{MÉTODOS}

Se realizó un estudio descriptivo, retrospectivo, transversal de una población total de pacientes con diagnóstico de úlcera venosa, valorados en una Unidad de atención especializada en el manejo de heridas durante el periodo de 01 de enero del 2017 y el 31 de agosto del 2019.

No se realizó cálculo muestral, porque fue incluida toda la población que cumplió con los criterios de inclusión.

Los criterios de inclusión fueron: personas mayores de 18 años, con ulcera por enfermedad venosa crónica con clasificación CEAP 5 o 6 y que hubiesen presentado por lo menos dos episodios de ulcera (recurrencia), mientras que se excluyeron aquellos pacientes con ulcera vascular mixta. 
Tabla 1. Clasificación CEAP $(\underline{5}, \underline{8})$.

\begin{tabular}{|c|c|c|c|}
\hline CLÍNICA (C) & $\begin{array}{l}\text { ETIOLOGÍA } \\
\text { (E) }\end{array}$ & $\begin{array}{l}\text { ANATOMÍA } \\
\text { (A) }\end{array}$ & $\begin{array}{l}\text { FISIOPATOLOGÍA } \\
\text { (P) }\end{array}$ \\
\hline $\begin{array}{l}\text { C0: No hay signos } \\
\text { visibles o palpables de } \\
\text { enfermedad venosa }\end{array}$ & EC : Congénita & $\begin{array}{l}\text { AS: Venas } \\
\text { superficiales }\end{array}$ & PR: Reflujo \\
\hline $\begin{array}{l}\text { C1: Presencia de } \\
\text { telangiectasias o venas } \\
\text { reticulares }\end{array}$ & EP: Primaria & $\begin{array}{l}\text { AD: Venas } \\
\text { profundas }\end{array}$ & PO: Obstrucción \\
\hline $\begin{array}{l}\text { C2: Presencia de } \\
\text { varices tronculares }\end{array}$ & $\begin{array}{l}\text { ES: } \\
\text { Secundaria }\end{array}$ & $\begin{array}{l}\text { AP: Sistema } \\
\text { perforante }\end{array}$ & $\begin{array}{l}\text { PRO: Reflujo y } \\
\text { obstrucción }\end{array}$ \\
\hline C3: Edema & & & $\begin{array}{l}\text { PN: Sin causa } \\
\text { identificable }\end{array}$ \\
\hline $\begin{array}{l}\text { C4: Cambios cutáneos } \\
\text { relacionados con la } \\
\text { patología venosa: }\end{array}$ & & & \\
\hline $\begin{array}{l}\text { 4a: Pigmentación, } \\
\text { eccema }\end{array}$ & & & \\
\hline 4b: & & & \\
\hline $\begin{array}{l}\text { Lipodermatoesclerosis, } \\
\text { atrofia blanca }\end{array}$ & & & \\
\hline $\begin{array}{l}\text { C5: Cambios cutáneos + } \\
\text { Úlcera cicatrizada }\end{array}$ & & & \\
\hline $\begin{array}{l}\text { C6: Cambios cutáneos + } \\
\text { Úlcera activa }\end{array}$ & & & \\
\hline
\end{tabular}

** Actualización de la clasificación CEAP.

Fuente: Tomado de: Carrasco Carrasco E. \& Díaz Sánchez S. Recomendaciones para el manejo de la Enfermedad Venosa Crónica en Atención Primaria. Semergen. 2015; 15 (2): $23 \quad$ - 28. https://www.semfyc.es/grupos/recomendaciones-para-el-manejo-de-la-enfermedad-venosa-cronica-en-atencionprimaria/

Análisis estadístico: La recolección de los datos estuvo a cargos de las 3 investigadoras pertenecientes al programa de clínica de heridas, quienes debían diligenciar una ficha de recolección de datos con las variables incluidas en el estudio. Esta se realizó a partir de la revisión de los registros clínicos de los pacientes con estos diagnósticos. La base de datos fue registrada en Excel y se analizó en el paquete estadístico SPSS. El análisis univariado se realizó determinando frecuencias absolutas y relativas en las variables categóricas, en el caso de las variables cuantitativas se calcularon medidas de tendencia central y medidas de dispersión según la distribución de la variable.

El principal sesgo que se puede presentar es el de selección, para lo cual se establecieron los criterios de inclusión y exclusión. 
Consideraciones éticas: El planteamiento de este estudio se ciñe a las normas internacionales y nacionales de ética en investigación. Basados en la Resolución 8430 de 1993 del Ministerio de Salud, se clasifica como una investigación sin riesgo (revisión de historial clínico, sin intervención a las personas. Al no haber ningún requerimiento de intervención y reconociendo que la historia clínica es un documento médico legal, se hizo la solicitud al Hospital San Rafael de la ciudad de Tunja, para que permitieran hacer la revisión de cada una de los registros clínicos de los pacientes con tales diagnósticos.

\section{RESULTADOS}

\section{Prevalencia de recurrencia.}

Durante el período se atendieron 140 pacientes con diagnóstico de úlcera vascular por enfermedad venosa, de los cuales 22 presentaron recurrencia de la patología, estimando una prevalencia de recurrencia del $15.7 \%$.

\section{Características sociodemográficas.}

22 pacientes presentaron recurrencia de la úlcera. La media de edad de los pacientes fue de 65 años con una desviación estándar de 13,8 años y coeficiente de variación de 21,3. En la tabla 2 se muestran las características sociodemográficas.

\section{Características clínicas.}

El índice de masa corporal promedio de la población fue de $28,6 \mathrm{~m} / \mathrm{kg} 2$ con una desviación estándar de $5,7 \mathrm{~ms} / \mathrm{kg} 2$. De acuerdo a los protocolos de tamizaje con la escala de Ferguson, el $22,7 \%$ de los pacientes presentaban desnutrición proteico-calórica según los registros de la historia clínica. El promedio de albumina sérica fue de $3.8 \mathrm{~g} / \mathrm{dL}, 2$ personas $(9 \%)$ presentaban niveles bajos de esta proteína.

Al 90,9\% se les realizó ultrasonido-doppler venoso como método diagnóstico, el porcentaje restante fue diagnosticado mediante las características clínicas de la lesión y la extremidad. Se encontró un predominio de afección en la pierna izquierda $(68.2 \%)$, seguido de la derecha $(22.7 \%)$ y bilateral $(9.1 \%)$.

El tiempo promedio de elevación de la extremidad fue de 50.9 minutos al día y, el promedio de tiempo en bipedestación fue de 6.6 horas al día.

La vena que más se vio afectada fue la safena mayor. El número de recurrencias de úlceras venosas varío entre 2 y 8 , con un promedio de 3.4 repeticiones, los cuales se presentaban con mayor frecuencia entre 3 a 6 meses y un año después del episodio. En la tabla 3 se muestran las características clínicas de los pacientes. 
Tabla 2. Características sociodemográficas.

\begin{tabular}{|c|c|c|c|c|}
\hline Variable & $\begin{array}{l}\text { Frecuencia } \\
(\mathrm{n}: 22)\end{array}$ & $\begin{array}{l}\text { Porcentaje } \\
(\%)\end{array}$ & IC 95\% & \\
\hline CARACTERISTICAS SOCIO & DEMOGRÁFI & & & \\
\hline Sexo & & & & \\
\hline Femenino & 16 & 72,7 & 54.12 & 91.34 \\
\hline Masculino & 6 & 27,3 & 8.664 & 45.88 \\
\hline Estado civil & & & & \\
\hline Casado & 8 & 36,4 & 16.26 & 56.46 \\
\hline Divorciado & 6 & 27,3 & 8.664 & 45.88 \\
\hline Viudo & 4 & 18,2 & 20.66 & 34.3 \\
\hline Soltero & 3 & 13,6 & 0.0 & 27.98 \\
\hline Unión libre & 1 & 4,5 & 0.0 & 13.25 \\
\hline Ocupación & & & & \\
\hline Labor en casa & 18 & 81,8 & 65.7 & 97.93 \\
\hline Auxiliar de cocina & 1 & 4,5 & 0.0 & 13.25 \\
\hline Auxiliar de enfermería & 1 & 4,5 & 0.0 & 13.25 \\
\hline Carpintero & 1 & 4,5 & 0.0 & 13.25 \\
\hline Tendero & 1 & 4,5 & 0.0 & 13.25 \\
\hline Estrato socioeconómico & & & & \\
\hline Bajo (1-2) & 13 & 59,1 & 38.55 & 79.63 \\
\hline Medio (3-4) & 9 & 40,9 & 20.37 & 61.45 \\
\hline Vive solo & & & & \\
\hline No & 17 & 77,3 & 59.76 & 94.78 \\
\hline $\mathrm{Si}$ & 5 & 22,7 & 5.217 & 40.24 \\
\hline Comorbilidades & & & & \\
\hline Hipertensión arterial & 16 & 72,7 & 54.12 & 91.34 \\
\hline $\begin{array}{l}\text { Sobrepeso } \quad\left({ }^{*} I M C \quad>25\right. \\
\left.\mathrm{m} / \mathrm{kgs}^{2}\right)\end{array}$ & 10 & 45,5 & 24.65 & 66.26 \\
\hline $\mathrm{EPOC}^{* *}$ & 6 & 27,3 & 8.664 & 45.88 \\
\hline $\begin{array}{l}\text { Desnutrición proteico- } \\
\text { calorica }\end{array}$ & 5 & 22,7 & 5.217 & 40.24 \\
\hline Antecedente de TVP ${ }^{* \star *}$ & 5 & 22,7 & 5.217 & 40.24 \\
\hline Tabaquismo & 5 & 22,7 & 5.217 & 40.24 \\
\hline Dislipidemia & 3 & 13,6 & 0.0 & 27.98 \\
\hline Fibrilación auricular & 2 & 9,0 & 0.0 & 21.1 \\
\hline Comunicación interauricular & 1 & 4,5 & 0.0 & 13.25 \\
\hline Hipertrofia septal & 1 & 4,5 & 0.0 & 13.25 \\
\hline
\end{tabular}


Tabla 3. Características clínicas de los pacientes.

\begin{tabular}{|c|c|c|c|c|}
\hline Variable & $\begin{array}{l}\text { Frecuencia } \\
(n: 22)\end{array}$ & $\begin{array}{l}\text { Porcentaje } \\
(\%)\end{array}$ & IC 95\% & \\
\hline \multicolumn{5}{|c|}{$\begin{array}{l}\text { CARACTERISTICAS CLINICAS } \\
\text { Hallazgos ecográficos }\end{array}$} \\
\hline $\begin{array}{l}\text { Enfermedad en safena } \\
\text { mayor }\end{array}$ & 8 & 36,4 & 16.26 & 56.46 \\
\hline $\begin{array}{l}\text { Enfermedad en safena } \\
\text { mayor y menor }\end{array}$ & 2 & 9,1 & 0.0 & 21.1 \\
\hline No se realizó & 2 & 9,1 & 0.0 & 21.1 \\
\hline $\begin{array}{l}\text { Enfermedad en safena } \\
\text { mayor, menor y perforantes }\end{array}$ & 1 & 4,5 & 0.0 & 13.25 \\
\hline $\begin{array}{l}\text { Enfermedad en safena } \\
\text { mayor, menor y profunda }\end{array}$ & 1 & 4,5 & 0.0 & 13.25 \\
\hline $\begin{array}{l}\text { Enfermedad en safena } \\
\text { mayor y perforantes }\end{array}$ & 1 & 4,5 & 0.0 & 13.25 \\
\hline $\begin{array}{l}\text { Enfermedad en safena } \\
\text { menor }\end{array}$ & 1 & 4,5 & 0.0 & 13.25 \\
\hline $\begin{array}{l}\text { Enfermedad en safena } \\
\text { menor y profunda }\end{array}$ & 1 & 4,5 & 0.0 & 13.25 \\
\hline $\begin{array}{l}\text { Insufiencia en venas } \\
\text { perforantes }\end{array}$ & 1 & 4,5 & 0.0 & 13.25 \\
\hline $\begin{array}{l}\text { Enfermedad } \\
\text { profunda }\end{array}$ & 1 & 4,5 & 0.0 & 13.25 \\
\hline Accesorias insuficientes & 1 & 4,5 & 0.0 & 13.25 \\
\hline $\begin{array}{l}\text { Enfermedad } \quad \text { venosa } \\
\text { superficial }\end{array}$ & 1 & 4,5 & 0.0 & 13.25 \\
\hline Enfermedad venosa crónica & 1 & 4,5 & 0.0 & 13.25 \\
\hline \multicolumn{4}{|l|}{ Tiempo de recurrencia } & 34.3 \\
\hline 3 a 6 meses & 7 & 31,8 & 12.36 & 51.28 \\
\hline 6 a 9 meses & 2 & 9,1 & 0.0 & 21.1 \\
\hline 9 a 12 meses & 2 & 9,1 & 0.0 & 21.1 \\
\hline Más de 12 meses & 7 & 31,8 & 12.36 & 51.28 \\
\hline
\end{tabular}

Fuente: Propio de los autores.

\section{DISCUSIÓN}

Se ha estimado que entre el 75 al $80 \%$ de las úlceras del miembro inferior se deben a etiología venosa, donde el $37 \%$ son recurrentes $(\underline{8}, \underline{9})$. Álvarez reportó las ulceras tienen un promedio de 3.8 recurrencias anualmente (10), la prevalencia de recurrencia fue menor en este estudio, probablemente asociado dos elementos importantes, por un lado la presencia de un grupo especializado en el manejo y seguimiento de esta población, sin embargo, también puede estar relacionado con la muestra de pacientes atendidos en la institución. 
La enfermedad venosa crónica es más frecuente en el sexo femenino, lo cual facilita la aparición de ulceras vasculares $(11,12)$. Márquez et al $(13)$ reporto que el promedio de edad de la población fue de 54 años, sin embargo, Karanicolic (14) menciono que en su su estudio fue de 63 años, siendo este último dato similar a lo encontrado.

Al igual que otros autores, se encontró que menos del $50 \%$ de las personas con esta entidad patológica se encuentran laborando, con nivel socioeconómico medio o bajo, y generalmente, viven solos $(\underline{15}, \underline{16})$.

Labrolopoulos (17), reportó que las personas con un IMC mayor de $25 \mathrm{~kg} / \mathrm{m} 2$ tienen una tendencia a cicatrizar con mayor lentitud comparado con la población que está dentro de límites normales (OR: 3,1; IC 95\%:1,06-8,83).

Según lo reportado por Haughey $L$ y cols $(\underline{18})$, se ha demostrado una asociación entre la desnutrición, deterioro en la cicatrización de la herida y la presencia de heridas crónicas. La falta de una nutrición adecuada permite la tendencia a la cronicidad de la herida debido a la falta de respuestas curativas apropiadas. Esto se ve particularmente en el envejecimiento de la población (18), por alteración de los procesos de regeneración celular y dificultad para la curación de las heridas $(\underline{19}, \underline{20})$.

Distintos investigadores han reportado que estos pacientes tienen una alta exposición a comorbilidades o factores que predisponentes a la enfermedad venosa tales como el tabaquismos, consumo crónico de alcohol, sobrepeso, diabetes mellitus, hipertensión arterial enfermedad pulmonar obstructiva crónica y dislipidemias (21, 22). Distintas investigaciones han demostrado que aquellas personas que se exponen al consumo de tabaco (OR: 1.99; IC95\%:1.16-3.42), hipertensión arterial (OR: 1.47; IC: 1.17-2.02), y diabetes mellitus (OR: 1.69, IC: 1.19-2.39) tienen más riesgo de recurrencia $(\underline{23}, \underline{24})$, puesto que estas comorbilidades se asocian con alteraciones del endotelio vascular, lo que facilita la fuga capilar, acumulación de fibrina, trombocitosis e inflamación, damnificando así la oxigenación de la piel y de los tejidos adyacentes $(\underline{25}, \underline{26})$.

Las ulceras venosas tienen una tiempo de curación largo, el 50\% cicatriza en los primeros 4 meses, $20 \%$ hasta los dos años y un $8 \%$ cicatriza por completo al cabo de cinco años (27). Además, las lesiones de más de $5 \mathrm{cms} 2$, con 12 meses de duración son factores de mal pronóstico de cicatrización (르).

En la mayoría de los casos se ha encontrado que la pierna más afectada es la izquierda, esto debido una disposición anatómica, puesto que la vena iliaca izquierda se encuentra situada entre la arteria iliaca y el cuerpo de la quita vértebra lumbar, generando compresión mecánica constante (29).

Distintos investigadores han coincidido en que la terapia compresiva es una medida terapéutica esencial para al manejo de la ulcera venosa, donde se prefieren usar presiones que oscilen entre los 20 y $40 \mathrm{mmHg}$ ( $\underline{30}$ - 33), esto basado en una revisión sistemática realizada por Brölmann (34), quien refiere que las presiones medias y altas tienen mejor efecto en sanación y control de recurrencia. Sin embargo, la dificultad para colocar las medias compresivas, obligan a la colaboración de parte de los familiares, siendo este dato interesante 
en la presente investigación pues un alto porcentaje no contaban con el apoyo en el domicilio, refiriéndolo como una causal del no uso de esta terapia.

A pesar que en el presente estudio se encontró que los humectantes eran las cremas que más se usan, aún no hay estudios que recomienden el uso de alguno en particular $(\underline{35}, \underline{36})$.

Dentro de los métodos para prevenir la presencia de enfermedad y ulceras venosas se debe evitar la permanencia de pie durante tiempos prolongados, usar ropa ajustada, elevar las extremidades y realizar ejercicio (1), esto asociado a que en los estaciones sedentarias o la bipedestación, facilita una sobrecarga de volumen en los miembros inferiores, generando lesión endotelial, de las valvas y de la pared vascular conllevando así a una enfermedad venosa $(\underline{37}, \underline{38})$. Es importante tener en cuenta que esta población puede presentar una percepción pobre o mala sobre la calidad de vida, por lo tanto es importante el manejo interdisciplinario para adaptar al paciente a todo tipo de condiciones $(\underline{38}, \underline{39})$.

Limitaciones: La principal limitación del estudio fue la muestra poblacional pequeña, lo que dificulta la toma de decisiones, sin embargo, se podría reproducir ampliando el tamaño de muestra; además es importante tener en cuenta que el carácter retrospectivo del estudio depende de la calidad de las historias clínicas realizadas.

\section{CONCLUSIONES}

La prevalencia de recurrencia de úlcera por enfermedad venosa crónica en usuarios del programa clínica de heridas del Hospital San Rafael de Tunja en el periodo 2017 a 2019, es menor que la reportada en la literatura, donde se ve afectada en mayor medida la población femenina. Casi la mitad de los pacientes con recurrencia presentaban obesidad, un cuarto de la población estaban en desnutrición. La afección se presentó con mayor frecuencia en la pierna izquierda. El número de recurrencias de úlceras venosas varío entre 2 y 8 , con un promedio de 3,4 repeticiones, los cuales se presentaban con mayor frecuencia entre 3 a 6 meses y un año después del episodio. El manejo quirúrgico y la terapia compresiva fueron las más realizadas, sin embargo, medidas como la elevación de la extremidad y realización de actividad física no se cumplen a cabalidad. Este estudio indica que hay una relación causaefecto de los factores de riesgo con la presencia de recidiva.

Este estudio nos aporta información valiosa, puesto que indica que la generación de grupos focalizados de atención a los pacientes, pueden mejorar las condiciones y disminuir el riesgo de complicaciones y/o secuelas, por lo que se sugiere desde estos resultados en las instituciones de atención de los pacientes la organización de programas como el de clínica de heridas.

Contribución de los autores: VABG, APBS, NJES, DCVQ: Conceptualización, investigación, curación de datos, escritura: preparación del borrador original, escritura: revisión y edición, visualización, supervisión, administración del proyecto. LJVR: Metodología, software, análisis formal y curación de datos, escritura: preparación del borrador original, escritura: revisión y edición.

Todos los autores han leído y aceptado la versión publicada del manuscrito.

Fondos: Esta investigación no recibió fondos externos. 
Conflictos de intereses: Los autores declaran no tener ningún conflicto de intereses.

\section{REFERENCIAS}

1. Adela Emilia Gómez Ayala. Úlceras vasculares. Factores de riesgo, clínica y prevención. 2008; 22 (6): 33-38. https://www.elsevier.es/es-revista-farmacia-profesional-3-articulo-ulcerasvasculares-factores-riesgo-clinica-13124067

2. Miquel Abbad C., Rial Horcajo R., Ballesteros Ortega M.D. \& García Madrid C. (2015) Guías de Práctica Clínica en Enfermedad Venosa Crónica. https://medes.com/publication/107046

3. Espejel Blancas J.A. et. (2018) Enfermedad venosa crónica y enfermedad hemorroidal en México. Revista México Angiología. 46(4): 204-212

4. Vázquez Hernández I. \& Acevedo Peña M. Prevalencia de enfermedad venosa periférica en el personal de enfermería. Enfermería Universitaria. 2016; 13(3):166-170.DOl: 10.1016/i.reu.2016.05.003

5. Carrasco Carrasco E. \& Díaz Sánchez S. Recomendaciones para el manejo de la Enfermedad Venosa Crónica en Atención Primaria. Semergen. 2015; 15 (2): 23 - 28. https://www.semfyc.es/grupos/recomendaciones-para-el-manejo-de-la-enfermedad-venosacronica-en-atencion-primaria/

6. Coccheri S., Mannello F. Development and use of sulodexide in vascular diseases: implications for treatment. Drug Design, development in therapy 2014; 4: 49-65. DOI: 10.2147/DDDT.S6762

7. Escudero J.R., Fernández F., Belmunt S. Prevalencia y características clínicas de la enfermedad venosa crónica en pacientes atendidos en Atención Primaria en España: resultados del estudio internacional Vein Consult Program. Cir Esp. 2014;92 (8):539-546. https://medes.com/publication/92815

8. Pannier F, Rabe E. Differential diagnosis of leg ulcers. Phlebology 2012; 28(1):55-0. DOl: $10.1177 / 0268355513477066$

9. World Health Organization. Management of substance abuse [Internet].[Consultado 2014 May 12]. http://www.who.int/substance abuse/research tools/whogolbref/en/

10. Rusbert Fernando Álvarez Del Río. Factores asociados a la Cicatrización de Úlceras Venosas de Miembros Inferiores y Calidad de Vida en Adultos, Medellín 2014-2015. Tesis. Facultad Nacional de Salud Pública Héctor Abad Gómez Medellín 2015. http://bibliotecadigital.udea.edu.co/handle/10495/3565

11. Secretaría de Salud. Guía de práctica clínica. Prevención, diagnóstico y tratamiento de la enfermedad venosa crónica. México: CENETEC; 2009

12. Espinóla CF, Bernau M, Aucejo M, et al. Prevalencia de várices en miembros inferiores en el personal del Hospital de Clínicas. Rev Chil Cir. 2007;59:342---7

13. Marques de Souza E, Bonetti Yoshida W, Aragao de Melo V, Bitencurt de Oliveir L. Ulcer Due to Chronic Venous Disease: A Sociodemographic Study in Northeastern Brazil Annals of Vascular Surgery. 2013;27:571-6. DOI: 10.1016/j.avsg.2012.07.021

14. Karanikolic V, Karanikolic A, Petrovic D, Stanojevic M. Prognostic factors related to delayed healing of venous leg ulcer treated with compression therapy. Dermatologica Sinica. 2015;33(4):206-9. DOI: 10.1016/i.dsi.2015.04.005

15. Moffatt CJ, Franks PJ, Doherty DC, Smithdale R, Martin R. Sociodemographic factors in chronic leg ulceration. Br J Dermatol 2006; 155(2):307-12. DOI: 10.1111/j.1365-2133.2006.07265.x

16. Gabriela Otero González*, Caroline Agorio Norstrom†, Miguel Martínez Asuaga. Úlceras de miembros inferiores Características clínico-epidemiológicas de los pacientes asistidos en la unidad de heridas crónicas del Hospital de Clínicas. Rev Méd Urug 2012; 28(3): 182-189. http://www.scielo.edu.uy/scielo.php?script=sci arttext\&pid=S1688-03902012000300004 
17. Labropoulos N, Wang E, Lanier S, Khan S. Factors Associated with Poor Healing and Recurrence of Venous Ulceration Plastic and Reconstructive Surgery 2012:179-86. DOI: 10.1097/PRS.0b013e3182362a53

18. Haughey L, Barbul A. Nutrition and Lower Extremity Ulcers: Causality and/or Treatment. Int J Low Extrem Wounds. 2017 Dec;16(4):238-243. DOI: 10.1177/1534734617737639.

19. Juárez-Cedillo, T. Changes in nutritional needs with aging. In: Watson, RR ed. Nutritional and Functional Foods for Healthy Aging. San Diego, CA: Academic Press; 2017:27-41.

20. Langer, G, Fink, A. Nutritional interventions for preventing and treating pressure ulcers. Cochrane Database Syst Rev. 2014;(6):CD003216. DOI: 10.1002/14651858.CD003216.pub2

21. I. Vázquez-Hernández $*$ y M. Acevedo-Pena. Prevalencia de enfermedad venosa periférica en el personal de enfermería. Enfermería Universitaria. 2016;13(3):166-170. DOl: 10.1016/.reu.2016.05.003

22. Vuylsteke M, Thomis S, Guillaume G, Modliszewski M, Weides N, Staelens I. Epidemiological study on chronic venous disease in Belgium and Luxembourg: prevalence, risk factors, and symptomatology. European Journal Of Vascular And Endovascular Surgery: The Official Journal Of The European Society For Vascular Surgery. 2015; 49(4):432-9. DOI: 10.1016/i.ejvs.2014.12.031

23. Vlajinac H, Marinkovic J, Maksimovic M, Radak D. Factors Related To Venous Ulceration: A Cross-Sectional Study. Angiology. 2014; 65(9):824-30. DOI: 10.1177/0003319713508218

24. Robertson L, Lee AJ, Gallagher K, Carmichael SJ, Evans CJ, McKinstry BH, et al. Risk factors for chronic ulceration in patients with varicose veins: A case control study. J Vasc Surg 2009; 49:1490-8. DOI: 10.1016/j.jvs.2009.02.237

25. Alguire $P$, Mathes B. Clinical manifestations of lower extremity chronic venous disease. Uptodate. 2014:1-15.

26. Ribu L, Birkeland K, Hanestadc B, Moumd T, Rustoen T. A longitudinal study of patients with diabetes and foot ulcers and theirhealth-related quality of life: wound healing and quality-of-life changes. Journal of Diabetes and Its Complications. 2008; 22:400-7. DOI: 10.1016/j.jdiacomp.2007.06.006

27. Kantor J, Margolis DJ. Management of leg ulcers. Semin Cutan Med Surg 2003; 22(3):212-21.

28. Nicolaides AN; Cardiovascular Disease Educational and Research Trust; European Society of Vascular Surgery; The International Angiology Scientific Activity Congress Organization; International Union of Angiology; Union Internationale de Phlebologie at the Abbaye des Vaux de Cernay. Investigation of chronic venous insufficiency: a consensus statement (France, March 5-9, 1997). Circulation 2000; 102(20):E126-63.

29. Lozano F. Origen del síndrome de la clase turista: John Homans 2016;68(3):255-256. DOI: 10.1016/i.cger.2013.01.006

30. Thomas D. Managing Venous Stasis Disease and Ulcers. Clinical geriatric medicine. 2013;29:415-24. DOl: 10.1016/j.cger.2013.01.006

31. Carreño Ávila P. Terapéutica de la Compresión en el tratamiento de la úlcera de etiología venosa. Todoheridas. 2010;1(1):4-16. http://scielo.isciii.es/scielo.php?script=sci arttext\&pid=S1134-928X2018000300153

32. Mauck K, Asi N, Elraiyah T, Undavalli C, Nabhan M, Altayar O, et al. Comparative systematic review and meta-analysis of compression modalities for the promotion of venous ulcer healing and reducing ulcer recurrence. J Vasc Surg. 2014;60(2, Supplement):71-90. 68. DOI: 10.1016/j.jvs.2014.04.060

33. Thomas F. O'Donnell Jr, MD, Marc A. Passman, MD, William A. Marston, MD, William J. Ennis, DO, Michael Dalsing, MD, Robert L. Kistner. Management of venous leg ulcers: Clinical practice guidelines of the Society for Vascular Surgery_ and the American Venous Forum. Journal vascular surgery. 2014; Volume 60, Number 2S: 3S-57S. DOI: 10.1016/.j.jvs.2014.04.049 
34. Brölmann FE, Ubbink DT, Nelson EA, Munte K, van der Horst CMAM, Vermeulen H. Evidencebased decisions for local and systemic wound care. Br J Surg 2012;99(9):1172- 83.

35. Dumville JC, Deshpande S, O'Meara S, Speak K. Hydrocolloid dressings for healing diabetic foot ulcers. The Cochrane database of systematic reviews. 2013;8:Cd009099. DOI: $10.1002 /$ bjs.8810

36. Palfreyman S, Nelson EA, Michaels JA. Dressings for venous leg ulcers: systematic review and

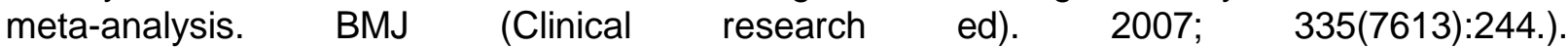
DOI: 10.1136/bmj.39248.634977.AE

37. Serra R, Grande R, Butrico L, et al. Effects of a new nutraceutical substance on clinical and molecular parameters in patients with chronic venous ulceration. Int Wound J 2016;13:88-96. DOI: 10.1111/iwj.12240

38. Raffetto JD. Pathophysiology of wound healing and alterations in venous leg ulcers- review. Phlebology 2016;31(1 Suppl):56-62. DOl: 10.1177/0268355516632998

39. Kurz X, Lamping D, Kahn S, Baccaglini U, Zuccarelli F, Spreafico G, et al. Do varicose veins affect quality of life? Results of an international population-based study. J Vasc Surg 2001; 34(4):641-8. DOl: 10.1067/mva.2001.117333 